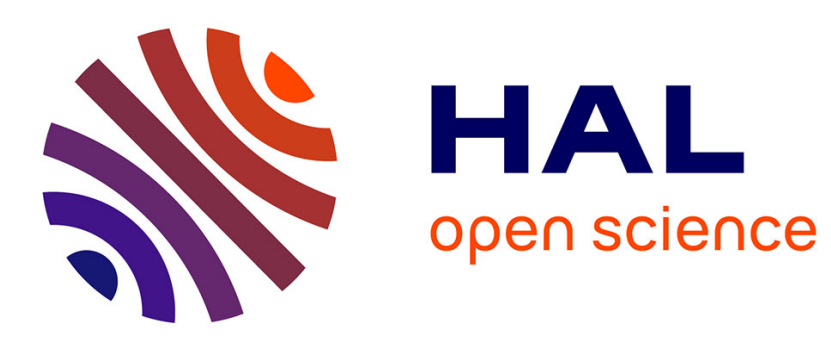

\title{
Nonlinear Adaptive Control of Networked Power Generators with Remote Measurement Units
}

\author{
Janailson Rodrigues Lima, Gilney Damm
}

\section{To cite this version:}

Janailson Rodrigues Lima, Gilney Damm. Nonlinear Adaptive Control of Networked Power Generators with Remote Measurement Units. 10th IEEE International Conference on Networking, Sensing and Control (ICNSC 2013), Apr 2013, Evry, France. pp.765-770, 10.1109/ICNSC.2013.6548834 . hal00786841

\section{HAL Id: hal-00786841 \\ https://hal.science/hal-00786841}

Submitted on 18 Mar 2014

HAL is a multi-disciplinary open access archive for the deposit and dissemination of scientific research documents, whether they are published or not. The documents may come from teaching and research institutions in France or abroad, or from public or private research centers.
L'archive ouverte pluridisciplinaire HAL, est destinée au dépôt et à la diffusion de documents scientifiques de niveau recherche, publiés ou non, émanant des établissements d'enseignement et de recherche français ou étrangers, des laboratoires publics ou privés. 


\section{Nonlinear Adaptive Control of Networked Power Generators with Remote Measurement Units}

\author{
Janailson Rodrigues Lima \\ SUPELEC \\ Gif-sur-Yvette, France \\ janailson.rodrigueslima@supelec.fr
}

\author{
Gilney Damm \\ Laboratoire IBISC \\ Université d'Evry Val d'Essone \\ Evry, France \\ gilney.damm@ibisc.fr
}

\begin{abstract}
A nonlinear adaptive control law for the stabilization of synchronous generators in a power network is designed, based on the new possibilities related with Smart Grids, in particular the insertion of remote measurements provided by Phasor Measurement Units (PMU). The proposed control system is designed in a robust way in order to cope with several uncertainties like the network parameters, and the intrinsic time delay on remote measurements. The proposed controller stability is rigorously proved, and computer simulations illustrate its good behavior, and suggest that the new measurement and data transmission devices can bring even better performance.
\end{abstract}

\section{INTRODUCTION}

Recently Smart Grids have become a very important trend in several research communities. Their new technological possibilities open the path to solve several hard problems in the literature. This paper aims exactly in investigating a new possibility on the control of networked power plants (power generators) using the rather new devices of Smart Grids known as Phasor Measurement Units (PMU). These devices are far beyond SCADA, and let one envisage the use of real time remote measurements on control schemes.

In the last three decades, the power systems have been growing in complexity and extension. The systems that were, at first, small and near the delivery point have spread to become interconnected, larger and more complex systems. One can see, for example, the evolution of the European system in [12]. This increase in complexity is important and necessary, and allows us to use the system at its full capability, but also makes the system more susceptible to disturbances and new phenomena, like inter-area oscillations ( [12]). In order to monitor these systems and acquire data to further studies, the measurement devices called Phasor Measurement Units are being widely developed and tested.

From the point of view of Control Engineering, the power systems urge for more modern and accurate techniques. Due to the new reality of power systems, the classic controllers: Automatic Voltage Regulator (AVR) and Power System Stabilizer (PSS) still largely used, are now decreasing in performance because the growing stress on the power systems' operation. The objective of the control of synchronous generator in a power system is to ensure Stability and Voltage Regulation even during network changes, failure and transient dynamics of others generators connected in the network. This task is even more complex due to non-linearity, parameter uncertainty and disturbances in the systems [11].

In [7] and [4] nonlinear adaptive laws are proposed, which achieve transient stabilization and voltage regulation, but the models neglect the perturbations from the transient state of others generators. Good results were obtained by studying a generator $g_{r}$ in a network of $N$ generators ( [5], [14]) and using the infinite bus model of a power network. In this context the adaptive law developed in [14], despite its complexity, guarantees transient stabilization and $\mathcal{L}_{2}$ and $\mathcal{L}_{\infty}$ disturbance attenuation using only local data and under reasonable assumptions. Several other works in the literature have addressed this field in the last decades ( [3], [9]) using different techniques, but without explicitly considering Smart Grids technologies.

In this work's main result, we studied the consequences of adding the measured data from PMUs in nonlinear adaptive laws developed following similar procedures than that in [14]. Even if the development and stability analysis are carried out in rigorous mathematical ways, controller robustness towards network parameters and data time delays are only illustrated by simulations. This procedure is a first step to verify the effectiveness of using these PMU for control, and to envisage future control schemes that will formally consider and deal with these disturbances, in particular the time delays.

First it was considered the insertion of undelayed measured data in the controller developed in [14] to check the performance gain remote measurements could provide compared to adaptations. It was then introduce continuing increasing delays to verify the robustness of the controller in respect to these time delays. Secondly, we propose a new nonlinear adaptive law considering the remote measurements. This control law performance is then illustrated in the simulation section face to real situations: short-circuit in a transmission line, interarea oscillation and large parameter changes in the network. We end up the work by some conclusions and future work that can be developed from the present results.

\section{Dynamic Model}

The dynamic model of a power systems of $N$ generators connected in a network is described by a $3 N$-order nonlinear model: 


$$
\begin{aligned}
\dot{\delta}_{i} & =\omega_{i} \\
\dot{\omega}_{i} & =-\frac{D_{i}}{2 H_{i}} \omega_{i}+\frac{\omega_{0}}{2 H_{i}} P_{m i}-\frac{\omega_{0}}{2 H_{i}} P_{e i} \\
\dot{E}_{q i}^{\prime} & =\frac{k_{c i}}{T_{d 0 i}^{\prime}} u_{f i}-\frac{E_{q i}^{\prime}}{T_{d 0 i}^{\prime}}-\frac{\left(x_{d i}-x_{d i}^{\prime}\right)}{T_{d 0 i}^{\prime}} I_{d i}
\end{aligned}
$$

and, for the $i$-th generator, we have the following electrical equations:

$$
\begin{aligned}
P_{e i}= & E_{q i}^{\prime} \sum_{j=1}^{n}\left[E_{q j}^{\prime} G_{i j} \cos \left(\delta_{i j}\right)+E_{q j}^{\prime} B_{i j} \sin \left(\delta_{i j}\right)\right] \\
= & E_{q i}^{\prime} I_{q i} \\
Q_{e i}= & E_{q i}^{\prime} \sum_{j=1}^{n}\left[E_{q j}^{\prime} G_{i j} \sin \left(\delta_{i j}\right)-E_{q j}^{\prime} B_{i j} \cos \left(\delta_{i j}\right)\right] \\
& -x_{d i}^{\prime}\left(I_{d i}^{2}+I_{q i}^{2}\right) \\
= & E_{q i}^{\prime} I_{d i}-x_{d i}^{\prime}\left(I_{d i}^{2}+I_{q i}^{2}\right) \\
I_{d i}= & \sum_{j=1}^{n}\left[E_{q j}^{\prime} G_{i j} \sin \left(\delta_{i j}\right)-E_{q j}^{\prime} B_{i j} \cos \left(\delta_{i j}\right)\right] \\
I_{q i}= & \sum_{j=1}^{n}\left[E_{q j}^{\prime} G_{i j} \cos \left(\delta_{i j}\right)+E_{q j}^{\prime} B_{i j} \sin \left(\delta_{i j}\right)\right] \\
\delta_{i j}= & \delta_{i}-\delta_{j} \\
V_{t i}= & \sqrt{\left(x_{d i}^{\prime} I_{q i}\right)^{2}+\left(E_{q i}^{\prime}-x_{d i}^{\prime} I_{d i}\right)^{2}}
\end{aligned}
$$

Where the first two equations of model 1 are the mechanical dynamic of the $i$-th generator involving the power angle $\delta_{i}(\mathrm{rad})$, the relative angular speed $\omega_{i}(\mathrm{rad} / \mathrm{s})$, the active electrical power $P_{e i}$ (p.u.), the input mechanical power $P_{m i}$ (p.u.)(seen in this work as a unknown input), the synchronous angular speed $\omega_{0}(\mathrm{rad} / \mathrm{s})$, the damping coefficient $D_{i}$ (p.u.)(often neglected) and the inertia constant $H_{i}$ (s). The others equations describe the electrical behaviour of the system, including disturbance, characteristics of the network and interactions between generators. In these equations are included the transient quadrature EMF $E_{q i}^{\prime}$ (p.u.), the control input $u_{f i}$ (p.u.) to the thyristor amplifier, the gain $k_{c i}$ of the excitation amplifier, the direct axis transient open circuit time constant $T_{d 0 i}^{\prime}(\mathrm{s})$, the direct axis reactance and the direct axis transient reactance $x_{d i}$ (p.u.) and $x_{d i}^{\prime}$ (p.u.), respectively, the reactive electrical power $Q_{e i}$ (p.u.), the direct and quadrature axis current $I_{d i}$ and $I_{q i}$, the terminal voltage $V_{t i}$ and the $G_{i j}$ (p.u.) and $B_{i j}$ (p.u.) which are the elements of the $i$-th row and $j$-th column of the conductance matrix $G$ and the susceptance matrix $B$. These two matrix represent the transmission network connecting the generators, they are changing as the load of any generator changes and are unmeasurable parameters.

$I_{d i}$ and $I_{q i}$ play an important role in this model, as one can see they are the interactions between generators. Within these equations there are some states of the remote generators, the power angle and the quadrature EMF, and parameters of the network, such as the susceptance and conductance. Furthermore these parameters are unmeasurable as they depend on the whole state of the power system which is constantly changing.

In normal operation, called pre-fault, the system has as equilibrium point:

$$
\begin{aligned}
\delta_{i} & =\delta_{i 0} \\
\omega_{i} & =0 \\
E_{q i}^{\prime} & =E_{q i 0}^{\prime}
\end{aligned}
$$

which implies

$$
P_{e i}=P_{m i}
$$

for $1<i<n$, that must guarantees that the output voltage $V_{t i}$ of each generator is equal to its reference constant value $V_{t i}^{*}, 1<i<n$.

As one can see, $P_{e i}$ appears linearly in the dynamics of the angular speed and is also an output to be controlled once it must be held equal to $P_{m i}$. Furthermore it is measurable (available for feedback) and using it would give to the system a triangular structure, making it easier to design a controller.

Considering constant $G_{r j}$ and $B_{r j}$, for $1<j<n$ and using equation 2 we can derive the dynamic of $P_{e r}$, the power output of the $r$-th generator:

$$
\begin{aligned}
\dot{P}_{e r}= & \dot{E}_{q r}^{\prime} I_{q r}+\dot{I}_{q r}^{\prime} E_{q r}=\dot{E}_{q r}^{\prime}\left[I_{q r}+E_{q r}^{\prime} G_{r r}\right] \\
& +E_{q r}^{\prime} \sum_{j=1, j \neq r}^{n}\left[\dot{E}_{q j}^{\prime} G_{r j} \cos \left(\delta_{r j}\right)+\dot{E}_{q j}^{\prime} B_{r j} \sin \left(\delta_{r j}\right)\right] \\
& -E_{q r}^{\prime} \sum_{j=1, j \neq r}^{n}\left[E_{q j}^{\prime} G_{r j} \sin \left(\delta_{r j}\right)-E_{q j}^{\prime} B_{r j} \cos \left(\delta_{r j}\right)\right]\left[\omega_{r}-\omega_{j}\right]
\end{aligned}
$$

This last equation can be rearranged and the dynamics of the system with this new state variable can be represented as:

$$
\begin{aligned}
\dot{\delta}_{r}= & \omega_{r} \\
\dot{\omega}_{r}= & -\frac{D_{r}}{2 H_{r}} \omega_{r}+\frac{\omega_{0}}{2 H_{r}} P_{m r}-\frac{\omega_{0}}{2 H_{r}} P_{e r} \\
\dot{P}_{e r}= & -\frac{1}{T_{d 0 r}^{\prime}} P_{e r}-\frac{\left(x_{d r}-x_{d r}^{\prime}\right)}{T_{d 0 r}^{\prime}} I_{d r} I_{q r}-\frac{G_{r r}\left(x_{d r}-x_{d r}^{\prime}\right)}{T_{d 0 r}^{\prime}} \frac{I_{d r} P_{e r}}{I_{q r}} \\
& -\frac{G_{r r}}{T_{d 0 r}^{\prime}} \frac{P_{e r}^{2}}{I_{q r}^{2}}+\left[\frac{\frac{k_{c r}}{T_{d 0 r}^{\prime}} I_{q r}^{2}+\frac{G_{r r} k_{c r}}{T_{d 0 r}^{\prime}} P_{e r}}{I_{q r}}\right] u_{f r} \\
& -\left(Q_{e r}+B_{r r} \frac{P_{e r}^{2}}{I_{q r}^{2}}+x_{d r}^{\prime}\left(I_{d r}^{2}+I_{q r}^{2}\right)\right) \omega_{r}+\frac{P_{e r}}{I_{q r}} R_{r}
\end{aligned}
$$

Where the term

$$
\begin{aligned}
R_{r}= & \sum_{j=1, j \neq r}^{n} \dot{\tilde{E}}_{q j}^{\prime}\left[G_{r j} \cos \left(\delta_{r j}\right)+B_{r j} \sin \left(\delta_{r j}\right)\right] \\
& +\sum_{j=1, j \neq r}^{n} \omega_{j}\left[E_{q j}^{\prime} G_{r j} \sin \left(\delta_{r j}\right)-E_{q j}^{\prime} B_{r j} \cos \left(\delta_{r j}\right)\right]
\end{aligned}
$$

represents the remote network dynamics, it means, the disturbance on the $r$-th generator caused by the remote generators out of their equilibrium points (as one can see, $R_{r}=0$ when in 
equilibrium). This expression of the network interconnection can not be measured, mainly because of the presence of $G_{r j}$, $B_{r j}$ and $\tilde{E}_{q j}^{\prime}=E_{q j}^{\prime}-E_{q j 0}^{\prime}, 1<j<n, j \neq r . R_{r}$ represents the main parameter requiring telecommunication of remote data, and as a consequence, plays a crucial role in this work.

We then define a new parametrization: $\theta_{1 r}=\frac{1}{T_{d 0 r}^{\prime}}, \theta_{2 r}=$ $\frac{\left(x_{d r}-x_{d r}^{\prime}\right)}{T_{d 0 r}^{\prime}}, \theta_{3 r}=\frac{G_{r r}\left(x_{d r}-x_{d r}^{\prime}\right)}{T_{d 0 r}^{\prime}}, \theta_{4 r}=\frac{G_{r r}}{T_{d 0 r}^{\prime}}, \theta_{5 r}=\frac{k_{c r}}{T_{d 0 r}^{\prime}}, \theta_{6 r}=$ $\frac{G_{r r} k_{c r}}{T_{d 0 r}^{\prime}}$ as the generator electrical constant parameters.

In practice, the exact values of some parameters are hard to obtain, specially $G_{r r}, B_{r r}$ and $P_{m r}$. This last one depends on the turbine mechanical dynamics and the load, which exact value is unknown. The first two parameters may change due to electrical failures and perturbations. In this work, we consider as known the parameters $\omega_{0}, H_{r}, D_{r}, T_{d r 0}^{\prime}, x_{d r}, x_{d r}^{\prime}$ and $k_{c r}$ which are mechanical or electrical parameters of the generator, and can be obtained by off-line experiments. In the same way, the unknown parameters are assumed to be within known positives bounds $\left(G_{r r m}, G_{r r M}\right),\left(B_{r r m}, B_{r r M}\right),\left(P_{m r m}, P_{m r M}\right)$ with $P_{m r}$ being a class $\mathcal{C}^{1}$ function and $\left|\dot{P}_{m r}(t)\right| \leq \dot{P}_{M r}$.

Physical considerations about load, transmission network and mechanical turbines make the considerations above reasonable in practical applications.

\section{Control Proposition}

In this chapter we develop our main result, a nonlinear adaptation law for the control of the synchronous generator based on the model described above and the design techniques found in [10] and [13] and developed in [14].

First we define the power angle and the relative angular speed errors as:

$$
\begin{aligned}
\tilde{\delta}_{r} & =\delta_{r}-\delta_{r s} \\
\tilde{\omega}_{r} & =\omega_{r}-\omega_{r}^{*}
\end{aligned}
$$

Where $\delta_{r s}$ is the power angle constant reference value (the pre-fault value) and $\omega_{r}^{*}$, the relative angular speed time-varying reference signal here defined as:

$$
\omega_{r}^{*}=-\frac{5}{4} k_{\delta r} \tilde{\delta}_{r}
$$

We define the active electrical power time-varying reference signal as:

$$
\begin{aligned}
P_{e r}^{*}= & \frac{2 H_{r}}{\omega_{0}}\left[\frac{5}{4} k_{\omega r} \tilde{\omega}_{r}+\frac{5}{4} k_{\delta r} \omega_{r}\right. \\
& \left.-\frac{D_{r}}{2 H_{r}} \omega_{r}^{*}+\tilde{\delta}_{r}+\frac{1}{k_{\omega p r}} \tilde{\omega}_{r}\right]+\hat{P}_{m r}
\end{aligned}
$$

Where $\hat{P}_{m r}$ is the estimate of the input mechanical power, obtained by the observer:

$$
\begin{aligned}
& \hat{P}_{m r}=\phi_{r}+\frac{2 H_{r}}{\omega_{0}}\left(\frac{5}{4} k_{p e r}+\frac{k_{r}}{4}+\frac{1}{k_{r}}+\frac{\omega_{0}^{2}}{16 H_{r}^{2}} k_{\omega p r}\right) \omega_{r} \\
& \dot{\phi}_{r}=\left(\frac{5}{4} k_{p e r}+\frac{k_{r}}{4}+\frac{1}{k_{r}}+\frac{\omega_{0}^{2}}{16 H_{r}^{2}} k_{\omega p r}\right)\left[-\phi_{r}+\frac{D_{r}}{\omega_{0}} \omega_{r}\right. \\
& \left.+P_{\text {er }}-\frac{2 H_{r}}{\omega_{0}}\left(\frac{5}{4} k_{p e r}+\frac{k_{r}}{4}+\frac{1}{k_{r}}+\frac{\omega_{0}^{2}}{16 H_{r}^{2}} k_{\omega p r}\right) \omega_{r}\right] \text { and: } \\
& \dot{\tilde{P}}_{m r}=-\left(\frac{5}{4} k_{p e r}+\frac{k_{r}}{4}+\frac{1}{k_{r}}+\frac{\omega_{0}^{2}}{16 H_{r}^{2}} k_{\omega p r}\right) \tilde{P}_{m r} \\
& +\dot{P}_{m r} \text {. }
\end{aligned}
$$

Finally, we can define the active electrical power tracking error:

$$
\tilde{P}_{e r}=P_{e r}-P_{e r}^{*}
$$

Now that the tracking error system is complete we may present the closed loop dynamic for the error system as:

$$
\begin{aligned}
\dot{\tilde{\delta}}_{r}= & -\frac{5}{4} k_{\delta r} \tilde{\delta}_{r}+\tilde{\omega}_{r} \\
\dot{\tilde{\omega}}_{r}= & -\left(\frac{5}{4} k_{\omega r}+\frac{D_{r}}{2 H_{r}}\right) \tilde{\omega}_{r}-\tilde{\delta}_{r}+\frac{\omega_{0}}{2 H_{r}} \tilde{P}_{m r}-\frac{\omega_{0}}{2 H_{r}} \tilde{P}_{e r} \\
& -\frac{1}{k_{\omega p r}} \tilde{\omega}_{r} \\
\dot{\tilde{P}}_{e r}= & -\frac{5}{4} k_{p e} \tilde{P}_{e r}+\frac{\omega_{0}}{2 H_{r}} \tilde{\omega}_{r}+\frac{P_{e r}}{I_{q r}} R_{r}-\frac{\tilde{G}_{r r}}{T_{d}^{\prime}} \frac{P_{e r}^{2}}{I_{q r}^{2}} \\
& -\tilde{G}_{r r} \frac{\left(x_{d r}-x_{d r}^{\prime}\right)}{T_{d}^{\prime}} \frac{I_{d r} P_{e r}}{I_{q r}}+\tilde{G}_{r r} \frac{P_{e r}}{\left(I_{q r}^{2}+\hat{G}_{r r} P_{e r}\right)} v_{r}^{\prime} \\
& -\tilde{B}_{r r} \frac{P_{e r}^{2}}{I_{q r}^{2}} \omega_{r}+\pi_{r}-\left(\frac{5}{4} k_{\omega r}+\frac{5}{4} k_{\delta r}+\frac{5}{4} k_{p e r}\right. \\
& \left.+\frac{k_{r}}{4}+\frac{1}{k_{r}}+\frac{\omega_{0}^{2}}{16 H_{r}^{2}} k_{\omega p r}+\frac{1}{k_{\omega p r}}\right) \tilde{P}_{m r}
\end{aligned}
$$

Where $v_{r}^{\prime}$ is equal to:

$$
v_{r}^{\prime}=\frac{\left(\theta_{5} I_{q r}^{2}+\hat{\theta}_{6} P_{e r}\right)}{I_{q r}} u_{f r}
$$

with $\hat{\theta}_{6}=\hat{G}_{r r} \theta_{5}\left(\theta_{5}\right.$ as defined in chapter 2). And also the robustifying term:

$$
\begin{aligned}
\pi_{r}= & -\frac{k_{R r}}{4} \frac{P_{e r}^{2}}{I_{q r}^{2}} \tilde{P}_{e r}-\frac{k_{r}}{4} \tilde{P}_{e r}\left[P_{e r}^{2}+I_{d r}^{2} I_{q r}^{2}+\frac{I_{d r}^{2} P_{e r}^{2}}{I_{q r}^{2}}\right. \\
& \left.+\frac{P_{e r}^{4}}{I_{q r}^{4}}\left(1+\omega_{r}^{2}\right)+\omega_{r}^{2}\left(I_{d r}^{2}+I_{q r}^{2}\right)^{2}\right]-\frac{k_{r}}{4}\left(\frac{5}{4} k_{\omega r}\right. \\
& +\frac{5}{4} k_{\delta r}+\frac{5}{4} k_{p e r}+\frac{k_{r}}{4}+\frac{1}{k_{r}}+\frac{\omega_{0}^{2}}{16 H_{r}^{2}} k_{\omega p r} \\
& \left.+\frac{1}{k_{\omega p r}}\right)^{2} \tilde{P}_{e r}-\frac{k_{r}}{4} \frac{P_{e r}^{2}}{I_{q r}^{2}} \tilde{P}_{e r} R_{r}^{2}
\end{aligned}
$$

and

$$
\tilde{P}_{m r}=P_{m r}-\hat{P}_{m r}
$$




$$
\begin{aligned}
v_{r}^{\prime}= & -\frac{5}{4} k_{p e} \tilde{P}_{e r}+\frac{\omega_{0}}{2 H_{r}} \tilde{\omega}_{r}+\frac{5 D_{r} k_{\delta r}}{4 \omega_{0}} \omega_{r}+\tilde{B}_{r r} \frac{P_{e r}^{2}}{I_{q r}^{2}} \omega_{r} \\
& +\tilde{G}_{r r}\left[\frac{\left(x_{d r}-x_{d r}^{\prime}\right)}{T_{d}^{\prime}} \frac{I_{d r} P_{e r}}{I_{q r}}+\frac{1}{T_{d}^{\prime}} \frac{P_{e r}^{2}}{I_{q r}^{2}}\right]+\frac{P_{e}}{T_{d}^{\prime}} \\
& +\frac{\left(x_{d r}-x_{d r}^{\prime}\right)}{T_{d}^{\prime}} I_{q r} I_{d r}+Q_{e} \omega_{r}+x_{d r}\left(I_{q r}^{2}+I_{d r}^{2}\right) \omega_{r} \\
& +\frac{5}{4} k_{\delta}\left(\hat{P}_{m r}-P_{e}\right)+\frac{2 H}{\omega_{0}} \omega \\
& +\frac{2 H}{\omega_{0}}\left[\frac{5}{4} k_{\omega r}+\frac{1}{k_{\omega p r}}\right]\left[\left(\frac{5}{4} k_{\omega r}+\frac{D_{r}}{2 H_{r}}\right) \tilde{\omega}_{r}+\tilde{\delta}_{r}\right. \\
& \left.+\frac{\omega_{0}}{2 H} \tilde{P}_{e r}+\frac{1}{k_{\omega p r}} \tilde{\omega}_{r}\right]+\pi_{r}
\end{aligned}
$$

As one may notice, the term of remote data $R_{r}$ appears in equation 12 , more precisely, in the term $\pi_{r}$. We suppose that the data to perform the calculation of this term will be measured by the PMU. These units allow us to collect remote information of others generators, knowing that we have, associated with $R_{r}$, a delay due data telecommunication.

Finally, we can define the Lyapunov Function for the system and apply equations 8 and 12 to obtain the dynamic of the adaptations $\hat{G}_{r r}$ and $\hat{B}_{r r}$ :

$$
\begin{aligned}
\mathcal{W}_{r}= & \frac{1}{2}\left(\tilde{\delta}_{r}^{2}+\tilde{\omega}_{r}^{2}+\tilde{P}_{e r}^{2}+\tilde{P}_{m r}^{2}\right) \\
& +\frac{1}{2} \beta_{B r r} \tilde{B}_{r r}^{2}+\frac{1}{2} \beta_{G r r} \tilde{G}_{r r}^{2}
\end{aligned}
$$

Where its time derivative is equals to:

$$
\begin{aligned}
\dot{\mathcal{W}}_{r}= & -\frac{5}{4} k_{\delta r} \tilde{\delta}_{r}^{2}-\left(\frac{5}{4} k_{\omega r}+\frac{D_{r}}{2 H_{r}}\right) \tilde{\omega}_{r}^{2}+\frac{\omega_{0}}{2 H_{r}} \tilde{P}_{m r} \tilde{\omega}_{r} \\
& -\frac{1}{k_{\omega p r}} \tilde{\omega}_{r}^{2}-\frac{5}{4} k_{p r} \tilde{P}_{e r}^{2}+\pi_{r} \tilde{P}_{e r}+\frac{P_{e r}}{I_{q r}} R_{r} \tilde{P}_{e r} \\
& -\left(\frac{5}{4} k_{\omega r}+\frac{5}{4} k_{\delta r}+\frac{5}{4} k_{p e r}+\frac{k_{r}}{4}+\frac{1}{k_{r}}+\frac{\omega_{0}^{2}}{16 H_{r}^{2}} k_{\omega p r}\right. \\
& \left.+\frac{1}{k_{\omega p r}}\right) \tilde{P}_{m r} \tilde{P}_{e r}+\dot{P}_{m r} \tilde{P}_{m r}-\tilde{B}_{r r}\left[\frac{P_{e r}^{2} \omega_{r} \tilde{P}_{e r}}{I_{q r}^{2}}\right. \\
& \left.+\beta_{B r r} \dot{\hat{B}}_{r r}\right]-\left(\frac{5}{4} k_{p e r}+\frac{k_{r}}{4}+\frac{1}{k_{r}}+\frac{\omega_{0}^{2}}{16 H_{r}^{2}} k_{\omega p r}\right) \tilde{P}_{m}^{2} \\
& -\tilde{G}_{r r}\left[\frac{\left(x_{d r}-x_{d r}^{\prime}\right)}{T_{d}^{\prime}} \frac{I_{d r} P_{e r}}{I_{q r}} \tilde{P}_{e r}+\frac{P_{e r}^{2} \tilde{P}_{e r}}{T_{d}^{\prime} I_{q r}^{2}}\right. \\
& \left.-\frac{P_{e r} v_{r}^{\prime} \tilde{P}_{e r}}{I_{q}^{2}+\hat{G}_{r r} P_{e r}}+\beta_{G r r} \dot{\hat{G}}_{r r}\right]
\end{aligned}
$$

So, using the projection algorithm as defined in [14], the dynamics for the two adaptations are

$$
\begin{aligned}
\dot{\hat{G}}_{r r}= & \operatorname{Proj}\left\{\frac { \tilde { P } _ { e r } } { \beta _ { G r r } } \left[-\frac{\left(x_{d r}-x_{d r}^{\prime}\right)}{T_{d}^{\prime}} \frac{I_{d r} P_{e r}}{I_{q r}}-\frac{P_{e r}^{2}}{T_{d}^{\prime} I_{q r}^{2}}\right.\right. \\
& \left.\left.+\frac{P_{e r} v_{r}^{\prime}}{I_{q}^{2}+\hat{G}_{r r} P_{e r}}\right]\right\} \\
\dot{\hat{B}}_{r r}= & \operatorname{Proj}\left(-\frac{\tilde{P}_{e r}}{\beta_{B r r}} \frac{P_{e r}^{2}}{I_{q r}^{2}} \omega_{r}\right)
\end{aligned}
$$

Back to equation 13, by completing the squares and using the proprieties of $\operatorname{Proj}(x)$, we can show that:

$$
\begin{aligned}
\dot{\mathcal{W}}_{r} \leq & -\frac{5}{4}\left(k_{\delta r} \tilde{\delta}_{r}^{2}+k_{\omega r} \tilde{\omega}_{r}^{2}+k_{p r} \tilde{P}_{e r}^{2}+k_{p e r} \tilde{P}_{m r}^{2}\right) \\
& +\frac{R_{r}(t)^{2}}{k_{R r}}
\end{aligned}
$$

which assure that all states are arbitrarily ultimately bounded, with the error region given by the disturbance $R_{r}(t)$ attenuated by the arbitrary design parameter $k_{R r}$. Furthermore, if no transmission errors or delay corrupt the measurements, and if we could exactly obtain the measurement of the interconnection expression $R_{r}(t)$, then we could obtain a globally exponentially stable system by erasing the last term in (15). In the real case where errors and delays are intrisical to the system, the equations above shows that the system will be ultimately bounded.

\section{Simulations}

To perform the simulation, the control developed here was applied at each generator of the well known Western System Coordinating Council (WSCC), a 3-machine, 9-bus system with parameters available in [2] and described as a two axis model:

$$
\begin{aligned}
\dot{\delta}_{i} & =\omega_{i} \\
\dot{\omega}_{i} & =-\frac{D_{i}}{2 H_{i}} \omega_{i}+\frac{\omega_{0}}{2 H_{i}} P_{m i}-\frac{\omega_{0}}{2 H_{i}} P_{e i} \\
\dot{E}_{d i}^{\prime} & =-\frac{E_{d i}^{\prime}}{T_{q 0 i}^{\prime}}-\frac{\left(x_{q i}-x_{d i}^{\prime}\right)}{T_{q 0 i}^{\prime}} I_{q i} \\
\dot{E}_{q i}^{\prime} & =\frac{k_{c i}}{T_{d 0 i}^{\prime}} u_{f i}-\frac{E_{q i}^{\prime}}{T_{d 0 i}^{\prime}}-\frac{\left(x_{d i}-x_{d i}^{\prime}\right)}{T_{d 0 i}^{\prime}} I_{d i}
\end{aligned}
$$

where we considered in the simulations, that $D_{i}=0$, for $1 \leq i \geq 3$. One may notice that this system is equivalent to the one described in chapter 2 by making $x_{q i}=x_{d i}^{\prime}$ neglecting, thus, the dynamic of the fast damper-winding $E_{d i}^{\prime}$.

The simulations were carried out to illustrate the perfor${ }^{r}$ mance of the controller during normal events, such as interarea oscillation and some intense events as a short-circuit in a transmission line. For this last one we compare the results with the controller in [14] with the same parameters described in the article.

(13) The inter-zone oscillation phenomenon was simulated by including, in the input mechanical power of one generator, a sinusoidal component with amplitude equals to $10 \%$ of the total input in low frequency, $1 \mathrm{~Hz}$. A rather soft, but constant phenomenon while the short-circuit is a fast and 
intense changing in the network and will show the controller robustness.

\section{A. Transmission Line Short-Circuit}

Here we present the effect of a three phase short circuit occurring at $t=1 \mathrm{~s}$. This is one of the hardest faults that can affect the system, but at the same time, a current fault that happens every day in average for a country wide system.

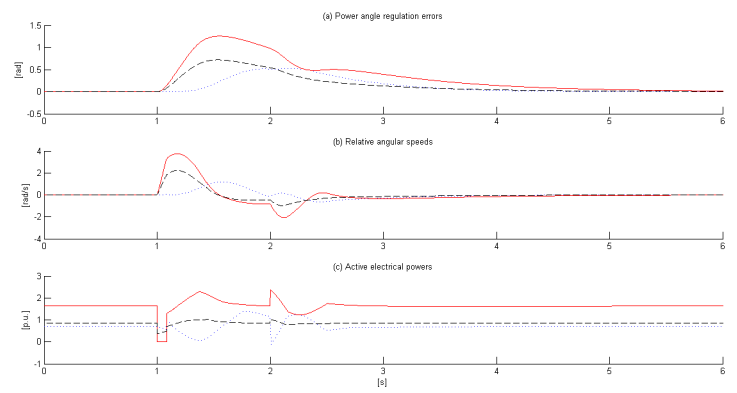

Fig. 1. Proposed controller [G1 (dot), G2 (solid), G3 (dash)]: a) $\delta_{i}-\delta_{i s}$; b) $\omega_{i}$; c) $P_{e i}$.

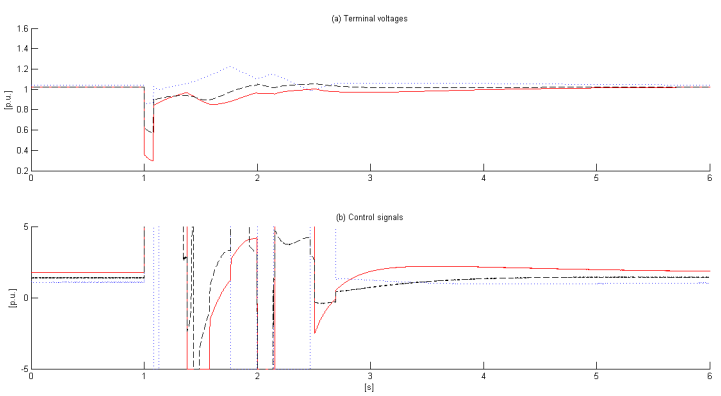

Fig. 2. Proposed controller [G1 (dot), G2 (solid), G3 (dash)]: a) $V_{t i}$; b) $u_{f i}$.

It can be seen in Fig 1, that the proposed controller allow all states of the three generators to be kept in reasonable bounds, and that they recover their original values from before the fault. In the same way the output voltage and the control input is shown in Fig 2.

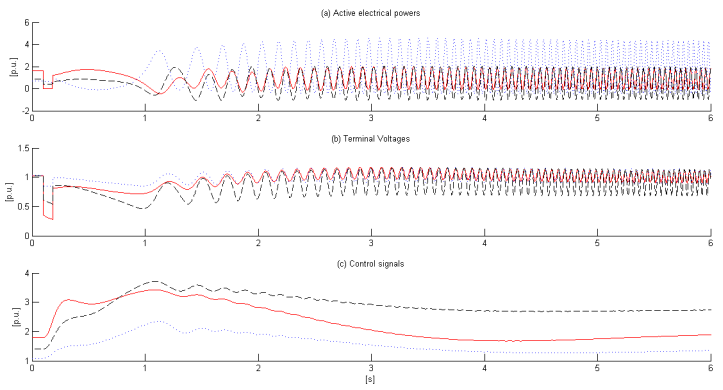

Fig. 3. Regulation with PSS [G1 (dot), G2 (solid), G3 (dash)]: a) $P_{e i}$; b) $V_{t i} ;$ c) $u_{f i}$.
For comparison, the same fault is dealt with a PSS (Power System Stabilizer) controller. As we can see in Fig 3, the controller cannot hold such fault.

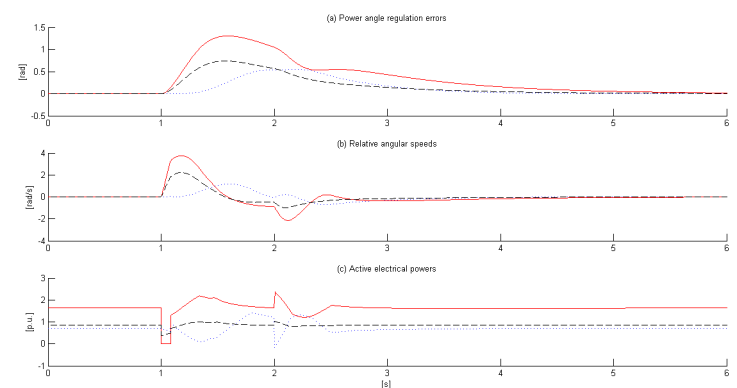

Fig. 4. Controller from [14] [G1 (dot), G2 (solid), G3 (dash)]: a) $\delta_{i}-\delta_{i s}$ ; b) $\omega_{i}$; c) $P_{e i}$

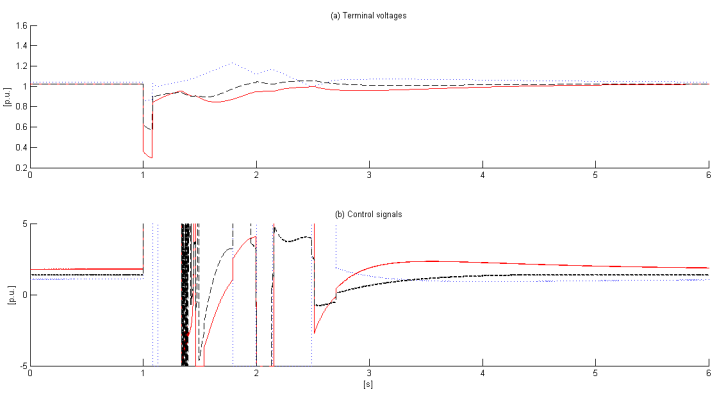

Fig. 5. Controller from [14] [G1 (dot), G2 (solid), G3 (dash)]: a) $V_{t i} ;$ b) $u_{f i}$.

Finally, the results for the controller proposed in [14] is presented in Fig 4 and Fig 5. In both groups of simulations, one can see that the proposed controller has very similar performances to [14], but with a simpler algorithm and with a smaller number of adaptations. Nevertheless both control algorithms managed to stabilize the system even in such critical situation, but the proposed algorithm is smoother which means less stress to the actuator.

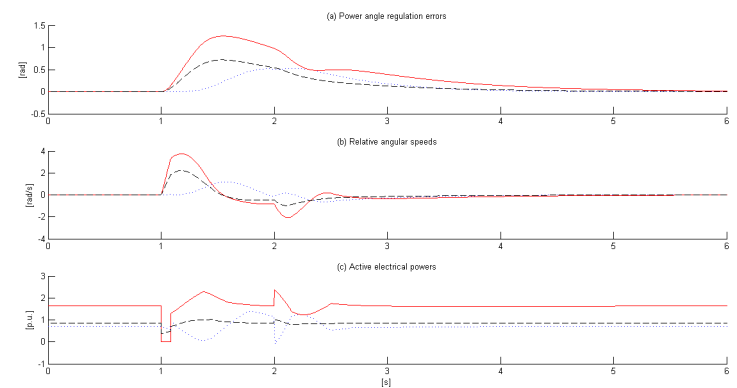

Fig. 6. Proposed controller [G1 (dot), G2 (solid), G3 (dash)]: a) $\delta_{i}-\delta_{i s}$; b) $\omega_{i}$; c) $P_{e i}$. 


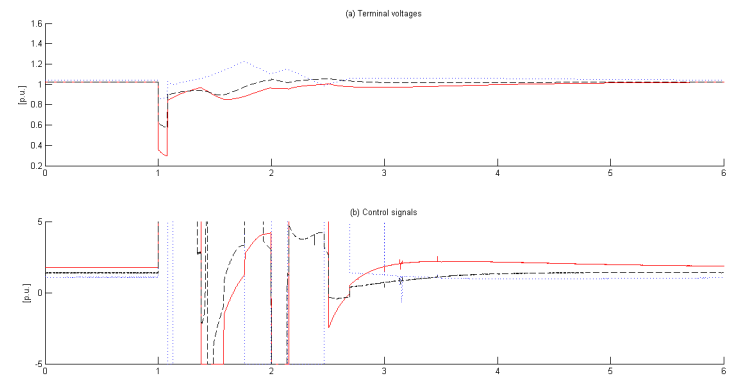

Fig. 7. Proposed controller [G1 (dot), G2 (solid), G3 (dash)]: a) $V_{t i}$; b) $u_{f i}$.

This last two figures (6 and 7) show the effects of adding a delay of $1 s$ in $R_{r}(t)$. The delay represents the data transmission time from the PMU to the local central. For nowadays technologies $1 s$ is quite a long delay, but shows that the effect of the data transmission is not relevant for the controller because the results were almost the same obtained by the controller without delay.

\section{B. Inter-area Oscillations}

In this section we will simulate the behaviour of the controller and the voltage output in the case of inter-zone oscillations.

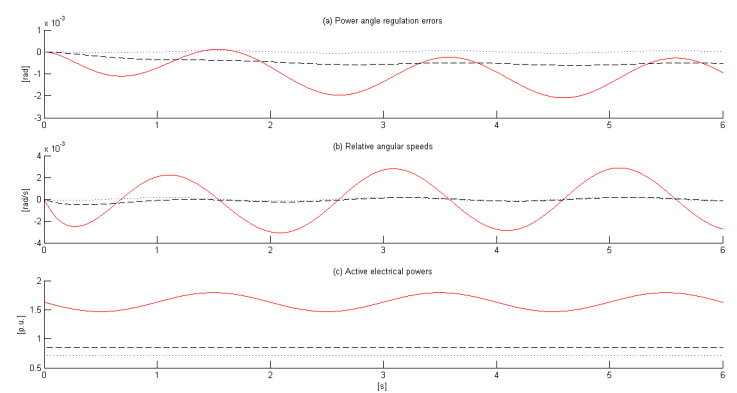

Fig. 8. Oscillation in G1 [G1 (dot), G2 (solid), G3 (dash)]: a) $\delta_{i}-\delta_{i s} ;$ b) $\omega_{i}$; c) $P_{e i}$

In Fig 8, we show the response of the controller for an oscillation in the active electrical power in generator 2. As one can see, we have small error, which shows a the response of the controller to such event.

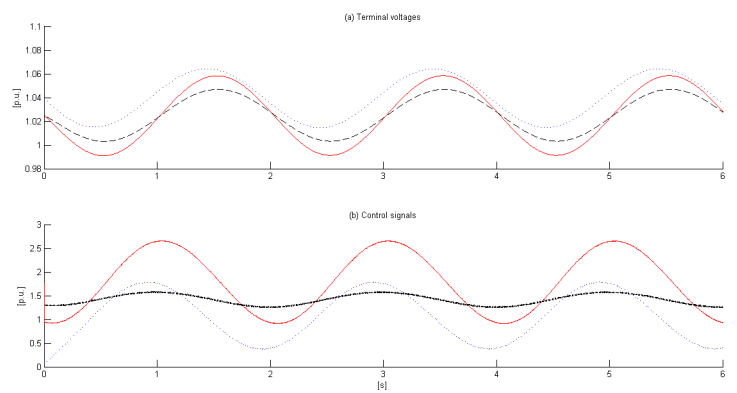

Fig. 9. Oscillation in G2 [G1 (dot), G2 (solid), G3 (dash)]: a) $V_{t i}$; b) $u_{f i}$.
In Fig 9 is the same scenario as Fig 8, but for the voltage output and the control signal. The control signal shows high amplitude oscillations in the same frequency of the mechanical power input. However, the output voltage remains stable in spite of the disturbance, with oscillations smaller than $5 \%$ of the current value.

\section{CONCLUSION}

The present work has developed a new control scheme inspired in previous works, in particular [14], but based in less adaptations and, with a simpler design. This new controller has also the advantage of being better fit for the use of remote measurements in its structure.

The good behaviour of the new controller can be appreciated in the proposed simulations, where the new controller was able to assure system's stability even face severe faults, and has shown to be as robust as the one in [14] even if much simpler.

In future works, we will continue to study how remote dynamics can be integrated to control schemes, and how time delays can be formally taken into account.

\section{REFERENCES}

[1] Escarela-Perez R. Espinosa-Perez G. Alvarez-Ramirez J., Cervantes I. A two-loop excitation control system for synchronous generators. International Journal of Electrical Power \& Energy Systems, 27(8):556-566, 2005.

[2] P. M. Anderson and A. A. Fouad. Power System Control and Stability. Wiley-IEEE Press: New York, 2003.

[3] A. Bazanella, A. S. Silva, and P. Kokotovic. Lyapunov design of excitation control for synchronous machines. In Proc. 36th IEEE - CDC, San Diego, CA, 1997. IEEE.

[4] Gilney Raymundo DAMM. Contributions to the Stabilization of Power Generators. PhD thesis, Universit Paris XI, november 2001.

[5] G.R. Damm, F. Lamnabhi-Lagarrigue, R. Marino, and C.M. Verrelli. Transient Stabilization and Voltage Regulation of a Synchronous Generator. ISTE- "Taming Heterogeneity and Complexity of Embedded Control, 2006.

[6] G.R. Damm, F. Lamnabhi-Lagarrigue, and C.M. Verrelli. Robust transient stabilization of a synchronous generator in an uncertain power network with transfer conductances. In European Control Conference 2009, Budapest, Hungary, August 23-26 2009.

[7] G.R. Damm, R. Marino, and F. Lamnabhi-Lagarrigue. Adaptive nonlinear output feedback for transient stabilization and voltage regulation of power generators with unknown parameters. Int. J. of Robust and Nonlinear Control, 14:833-855, 2004.

[8] Florian Dorfler and Francesco Bullo. Synchronization and transient stability in power networks and non-uniform kuramoto oscillators. IEEE Transactions on Automatic Control, pages 930 - 937, July 2010.

[9] L. Gao, L. Chen, Y. Fan, and H. Ma. A nonlinear control design for power systems. Automatica, 28, No. 5:975-979, 1992.

[10] H. Khalil. Nonlinear Systems. Prentice Hall, New Jersey, 3rd edition, 1996.

[11] P. Kundur. Power System Stability and Control. New York: McGrawHill, 1994.

[12] J. Lehner, M. Kaufhold, M. Treuer, and T. Weissbach. Monitoring of inter-area oscillations within the european interconnected network based on a wide area measuring system. April 2010.

[13] Riccardo Marino and Patrizio Tomei. Nonlinear Control Design: Geometric, Adaptive and Robust. Prentice Hall, 1st edition, 1995.

[14] C. M. Verrelli and G. Damm. Robust transient stabilization problem for a synchronous generator in a power network. International Journal of Control, 83(4), August 2010. 